\title{
Secondary Spectrum Access in TV-Bands with Combined Co-Channel and Adjacent Channel Interference Constraints
}

\author{
Lei Shi, Ki Won Sung, and Jens Zander \\ KTH Royal Institute of Technology, Wireless@KTH, Stockholm, Sweden \\ E-mail: 1shi@kth.se, sungkw@kth.se,jenz@kth.se
}

\begin{abstract}
The potential of VHF/UHF band as a candidate for secondary spectrum access, so called "TV white spaces", has been intensively investigated in recent years. However, the impact of the accumulated interference from multiple secondary users on different adjacent channels has not been well studied thus far, let alone the effect of combined interference from both co-channel and adjacent channels. This paper presents a framework for assessing secondary spectrum reuse opportunities for portable and mobile devices that comply with geo-location database concepts. The opportunity is evaluated in terms of the maximal number of secondary users that can access the "TV white space" simultaneously. Particular emphasis is given to the protection of TV receiver from harmful aggregate interference originated from not only the secondary users outside the TV coverage on the same channel but also those close to the TV receivers operating on different adjacent TV channels. An optimization problem is solved to maximize the number of secondary users admitted to the available TV channels at different locations. Through in-depth analysis of the interference characteristics of the optimal solution, it is identified that the cumulative effect of adjacent channel interferences has the dominant impact on TV reception, particularly for the case of secondary devices with limited transmit power. This suggests the possibility to achieve near-optimal exploitation of TV-bands for secondary reuse without explicit coordination of co-channel interference from the secondary users deployed over a wide geographical area.
\end{abstract}

Index Terms-TV White Space, secondary spectrum reuse, optimization, aggregate interference, adjacent channel interference, geo-location database.

\section{INTRODUCTION}

The rapidly growing demand for wireless services has driven the wireless industry to search for new radio spectrum to accommodate the enormous mobile data traffic. As a promising alternative to the lengthy spectrum reallocation process, secondary access to the locally or temporally underutilized licensed spectrum has attracted wide interests from industry and academic alike. Among the potential spectrum for secondary access, the VHF/UHF TV band is considered as the most promising candidate, thanks to its well defined primary usage and the favorable propagation characteristics for wide coverage and indoor penetation [1] [2].

The regulators have taken the initiative to establish regulatory framework for secondary access in TV white space (TVWS) [3] [4] [5]. These works have introduced the guideline for the secondary user (SU) to detect available TV channels and control its interference to the primary user (PU), using geo-location database or spectrum sensing. However, the discussions within the regulatory framework on secondary access in TV-bands has been primarily limited to single secondary user case so far.

Recent researches have extended the study to multipleSU case, developing both heuristic algorithm [7] [8] and optimized solutions [9] [10] for the secondary transmit power allocation. These studies have clearly demonstrated the impact of aggregate interference from multiple SUs on the secondary spectrum reuse opportunity, but their focus has been on the co-channel interference (CCI) from the SUs located outside the TV coverage area with long link-distance.

On the other hand, the TV receiver can be very vulnerable to adjacent channel interferences (ACI) as well, especially when the SUs are transmitting in the proximity of the victim receiver [11]. In [12], it has been shown that the ACI is the limiting factor for the secondary transmit power allocation with the method proposed in ECC report 159 [5]. In multipleSU scenario, particularly with dense deployment of portable or mobile secondary devices, the impact of ACI on TV reception is further signified by the cumulative effect of interferences from multiple channels [13]. A stochastic model for the accumulated ACI from multiple short range SUs transmitting on different channels has been proposed in [14], where the results also strengthened the importance of ACI in 'TV white space (TVWS)' analysis.

The method to combine these two types of interferences into a single constraint, however, is still lacking. The existing frameworks [5] [9] [14] have so far considered CCI and ACI separately, which could potentially lead to either inefficient secondary spectrum utilization, or overestimation of the spectrum reuse opportunity and cause excessive interference to the TV receivers.

The aim of this paper, therefore, is to propose a new framework for assessing the spectrum reuse opportunity in TV-bands for multiple portable/mobile secondary devices in compliance with the combined interference constraint. More specifically, we will optimize the number of admitted SUs on each available TV channel at different locations such that the total number of the active SUs is maximized. To our best knowledge, it is the first framework for the optimal 


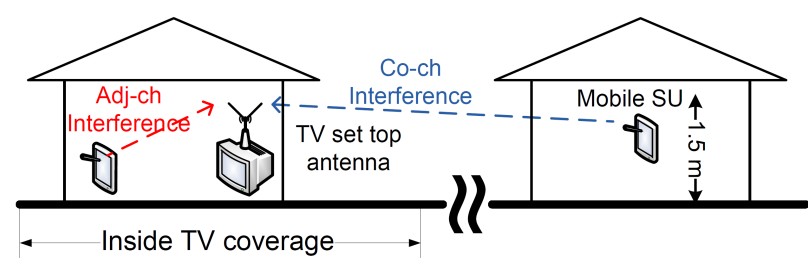

(a) Case A: indoor $\mathrm{SU}$ and set top TV reception antenna

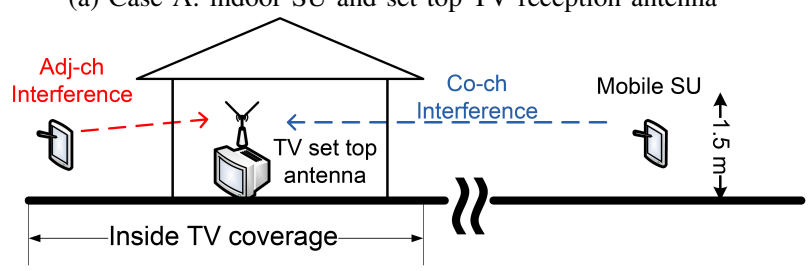

(b) Case B: outdoor SU and set top TV reception antenna

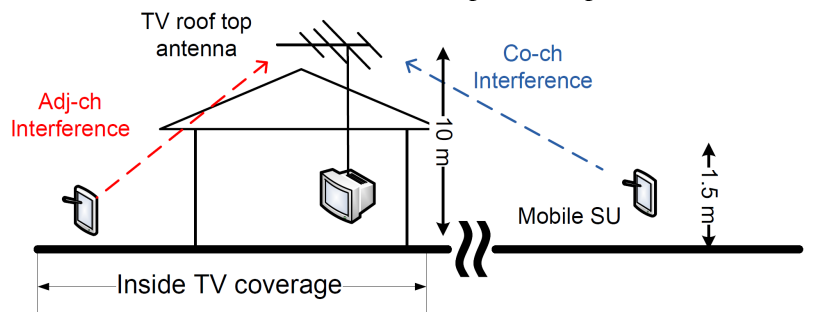

(c) Case $\mathrm{C}$ : indoor $\mathrm{SU}$ and roof top $\mathrm{TV}$ reception antenna

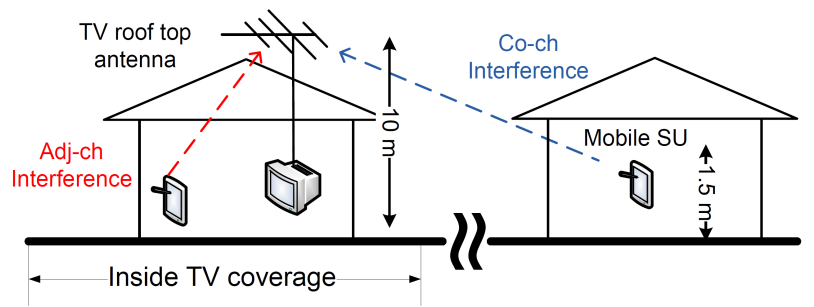

(d) Case D: outdoor SU and roof top TV reception antenna

Fig. 1: Secondary access scenarios

'TVWS' utilization with a comprehensive interference model that combines CCI from SUs outside the TV coverage and ACI from SUs close to the TV receivers. The optimal solutions are analyzed to identify whether ACI or CCI has the dominant damaging impact on the TV reception, so that we can provide more insights to improve the efficiency of the regulatory rule for secondary access in TV-bands.

In the following of this paper, we will start with a brief description of the studied scenario in Section II. The system model is explained in Section III. Then the optimization problem is formulated in Section IV, followed by numerical results and discussions in Section V. Finally conclusions are drawn in Section VI.

\section{SeCondary AcCess Scenario}

\section{A. Primary System}

In terrestrial TV broadcasting network, a TV station can cover an area with radius of $30-50 \mathrm{~km}$. The TV receives the signal through either a rooftop antenna or an indoor set-top antenna.
In order to avoid mutual interferences and to provide regional content, neighboring TV stations (or neighboring single frequency networks) typically transmit on different subsets of TV channels. Therefore, certain TV channels are locally unoccupied by the primary service and potentially available for secondary reuse.

\section{B. Secondary System}

As pointed out in [15], the commercial 'sweet point' for secondary reuse of 'TVWS' lies in the scenario with dense deployed short-range or indoor wireless system. Thus, the secondary system considered in our study is the portable or mobile devices, with limited transmit power and below clutter antennas. These devices could be regarded as the access point/user equipment in a 'WiFi-like' or 'Femtocelllike' secondary system. The SU is assumed to be randomly deployed either indoor or outdoor, and transmitting with fixed power, $p_{s u}$, on one of the locally unoccupied TV channels.

\section{Coexistence Deployment Scenarios}

With the above mentioned primary and secondary systems, different combinations of the coexistence scenarios are illustrated in Fig.1.

In both case A and case $\mathrm{B}$, the TV receiver is connected to an indoor set-top antenna. It can be expected that the impact of $\mathrm{ACI}$ on $\mathrm{TV}$ reception is stronger in case $\mathrm{A}$, as both the $\mathrm{SU}$ and the TV receiver are deployed indoor.

In case $\mathrm{C}$, the $\mathrm{TV}$ receiver is connected to a roof-top antenna while the SUs are deployed outdoor. Both links are free from wall penetration loss. The CCI link is an over-the-rooftop link, and will suffer less propagation loss as compared to the CCI link in case B, where both TV antenna and SU are below clutter height. In contrast, the ACI link is relatively weaker in case $\mathrm{C}$, because the closest $\mathrm{SU}$ is most likely to be outside the main lobe of the directional rooftop antenna pointed horizontally or even slightly upwards.

Case D is similar to case $\mathrm{C}$, as both links are subject to wall penetration loss. Hence, if we can identify that ACI has dominant effect in either case $\mathrm{C}$ or case $\mathrm{D}$, then the same conclusion can be readily applied to all the other cases. In the following of the study, we will focus on Case C, with rooftop directional antenna and outdoor SUs, as it is more comparable with one of the reference geometries described in [5].

\section{System Model}

\section{A. Secondary Access using Geo-location Database}

Following the suggestion by ECC [5], we adopts the geolocation database approach to regulate the secondary access to TV-bands, and protect the TV reception from harmful interference.

Given the limited resolution of the database and location detection capability of the $\mathrm{SU}$, it is impossible to estimate the reception quality of each individual TV receiver. Thus, the studied area is discretized into small area elements, denoted as 'pixel'. The geo-location database stores information for 
each area elements, such as the TV coverage quality, terrain elevation, population density, etc.

For notation purpose, we define the set of pixels over the entire studied region as $\mathcal{A}:=\left\{a_{1}, \ldots, a_{m}\right\}$. The set of TV channels is defined by $\mathcal{F}:=\left\{f_{1}, \ldots, f_{n}\right\}$. The TV signal power level on channel $f_{k}$ at pixel $a_{i}$ is denoted $S_{i}^{k}$. With shadow fading assumption, $S_{i}^{k}$ follows log-normal distribution with parameters $\mu_{S_{i}^{k}}$ and $\sigma_{S_{i}^{k}}$. Then, pixel $a_{i}$ is regarded as inside the coverage of channel $f_{k}$, if

$$
q_{1, i}^{k}=\operatorname{Pr}\left\{\frac{S_{i}^{k}}{N_{t v}} \geq \gamma_{0}\right\} \geq q_{1}^{*},
$$

where $N_{t v}$ is the TV receiver noise power plus TV selfinterference. $\gamma_{0}$ is the minimum signal-to-interference-andnoise ratio for successful TV reception. $q_{1}^{*}$ is the minimum required location probability without secondary interference, defined by the regulator as the measure for the primary system quality of service requirement.

A channel is regarded as occupied at a pixel if that pixel is inside its coverage, otherwise it is considered as available for local secondary access. The set of occupied TV channels in pixel $a_{i}$ is parameterized by an index set $\mathcal{K}_{i}$, i.e., $\mathcal{F}_{i}:=$ $\left\{f_{k} \mid k \in \mathcal{K}_{i}\right\}$. Similarly, the set of available TV channels in pixel $a_{i}$ is defined as $\mathcal{F}_{i}^{-}:=\left\{f_{\ell} \mid \ell \in \mathcal{L}_{i}\right\}$. We also define the index sets for the pixels inside and outside the coverage area of channel $f_{k}$ as $\mathcal{I}^{k}$ and $\mathcal{J}^{k}$, respectively.

An occupied TV channel must be protected from harmful secondary interference, with the following requirement:

$$
q_{2, i}^{k}=\operatorname{Pr}\left\{\frac{S_{i}^{k}}{N_{t v}+I_{i}^{k}} \geq \gamma_{0}\right\} \geq q_{2}^{*}
$$

where $I_{i}^{k}$ is the received secondary interference and $q_{2}^{*}$ is the minimum location probability under secondary interference defined by the regulator. Otherwise, the TV reception on that channel is deemed as being violated.

All the TVs in the same pixel $a_{i}$ receiving on the same channel $f_{k}$, are assumed to experience statistically the same level of TV signal power $S_{i}^{k}$ and secondary interference power $I_{k}^{i}$.

\section{B. Aggregate Interference Model}

Due to the imperfection of the TV receiver filter characteristic, the interferences from SUs that are simultaneously active on both co-channel and different adjacent channels would cause cumulative damaging effect on the TV reception. This cumulative effect of interferences from multiple channels can be modeled by the weighted summation of the interferences from different channels [13] (similar to the 'Nuisance Field' definition in [5]). Letting $I_{\mathrm{eff}, i}^{k}$ denotes the effective aggregate interference, we can define it as

$$
I_{\mathrm{eff}, i}^{k}=\sum_{t=1}^{N} \frac{\gamma_{k, \ell_{t}}}{\gamma_{0}} p_{s u} g_{d}\left(d_{t}\right) g_{\theta}\left(\theta_{t}\right) g_{f} .
$$

Here $N$ is the total number of active SUs. $\gamma_{k, \ell}$ is the minimum required TV signal to SU interference ratio between channel

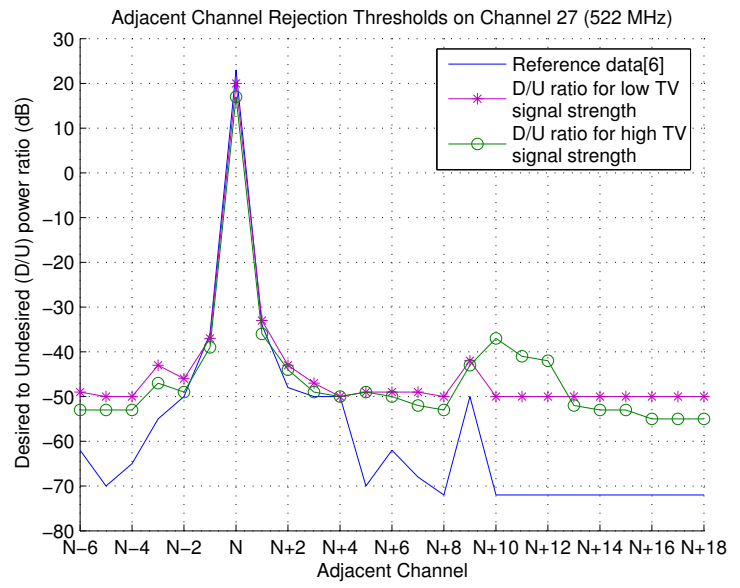

Fig. 2: Protection ratio as a function of frequency offset between TV signal and interfering signal [13].

$f_{k}$ and channel $f_{\ell}$ (also known as protection ratio [6]; see Fig. 2). $g_{d}(d)$ is the distance based attenuation. $g_{\theta}(\theta)$ is the receiver antenna gain, with incident angle $\theta$. And $g_{f}$ is the channel fading gain.

This aggregate interference model incorporates CCI and ACI from different channels. Nevertheless, in order to capture the distinguishing characteristics between the link geometries of $\mathrm{ACI}$ and $\mathrm{CCI}$, we re-write (3) as

$$
I_{\mathrm{eff}, i}^{k}=C C I_{i}^{k}+A C I_{i}^{k},
$$

where $C C I_{i}^{k}$ represents the aggregate co-channel interference, and $A C I_{i}^{k}$ the effective adjacent channel interferences.

1) Co-Channels Interferences: The CCI from a single active SU in pixel $a_{i}$ to the victim TV in pixel $a_{j}$ on the same channel $f_{k}$ is defined as

$$
c c i_{i, j}^{k}=p_{s u} g_{d}\left(d_{i, j}\right) g_{\theta}\left(\theta_{i, j}\right) g_{f},
$$

where $d_{i, j}$ is typically much larger than the pixel size. So it can be approximated by the distance between the center of the two pixels.

With slow fading assumption, $c c i_{i, j}^{k}$ can be modeled as a log-normal random variable (RV). We can express its first two cumulants [16] as

$$
\begin{gathered}
\kappa_{1}\left(c c i_{i, j}^{k}\right)=\exp \left[\mu_{c c i_{i, j}^{k}}+\sigma_{c c i_{i, j}^{k}}^{2} / 2\right], \\
\kappa_{2}\left(c c i_{i, j}^{k}\right)=\left[\exp \left(\sigma_{c c i_{i, j}^{k}}^{2}\right)-1\right] \exp \left(2 \mu_{c c i_{i, j}^{k}}+\sigma_{c c i_{i, j}^{k}}^{2}\right),
\end{gathered}
$$

where the location parameter, $\mu_{c c i}^{k} i_{i, j}^{k}$, can be directly estimated from the geo-location database

$$
\mu_{c c i_{i, j}^{k}}=\mathrm{E}\left[\ln \left(c c i_{i, j}^{k}\right)\right]=\ln \left(p_{s u} g_{d}\left(d_{i, j}\right) g_{\theta}\left(\theta_{i, j}\right)\right),
$$

and the scale parameter, $\sigma_{c c i_{i, j}^{k}}^{2}$, is the shadow fading variance in natural logarithmic scale.

In multiple-SU case, all CCI links between pixel $a_{i}$ and pixel $a_{j}$ are assumed to have the same distance and highly 
correlated shadow fading. Assuming the number of admitted SUs on channel $f_{k}$ in pixel $a_{j}$ being $x_{j}^{k}$, the aggregate CCI can be written as

$$
C C I_{i}^{k} \approx \sum_{j \in \mathcal{J}^{k}} x_{j}^{k} \cdot c c i_{i, j}^{k}
$$

Thus the $m$ th cummulants of $C C I_{i}^{k}$ are given as

$$
\kappa_{m}\left(C C I_{i}^{k}\right)=\sum_{j \in \mathcal{J}^{k}} x_{j}^{k} \cdot \kappa_{m}\left(c c i_{i, j}^{k}\right) .
$$

2) Adjacent Channels Interferences: Contrary to the CCI case, the SUs interfering on channels adjacent to the broadcasting TV channels are located in the proximity of the TV receiver, and the aggregate $\mathrm{ACI}$ is mainly dominated by a few SUs close by. Thus, we can limit the area of interference aggregation to the dominant interference region [17], from where $(100-\varepsilon) \%$ of the aggregate interference are originated.

Denoting $\Omega_{i}^{\ell}$ as the set of active SUs on channel $f_{\ell}$ inside the dominant interference region concentric to pixel $a_{i}$, we can express the aggregate ACI from SUs on different locally available TV channels, $\mathcal{L}_{i}$, as

$$
A C I_{i}^{k} \approx \sum_{\ell \in \mathcal{L}_{i}} \frac{\gamma_{k, \ell}}{\gamma_{0}} \sum_{t \in \Omega_{i}^{\ell}} p_{s u} g_{d}\left(d_{t}\right) g_{\theta}\left(\theta_{t}\right) g_{f} .
$$

According to our study [14] and the numerical results in [17], the radius of the dominant interference region is around 500 meters for $\epsilon=0.5$ in suburban environment. Considering the limited resolution of the geo-location database, we can assume the admitted SUs are uniformly distributed with constant density inside the dominant interference region. Thus, we have

$$
\lambda_{\Omega_{i}^{\ell}} \approx \lambda_{i}^{\ell}=\frac{x_{i}^{\ell}}{\left|a_{i}\right|}
$$

where $\left|a_{i}\right|$ is the size of pixel $a_{i}$.

Given the variance of the shadow fading and the user distribution density, the aggregate $\mathrm{ACI}$ can also be approximated by a log-normal RV [18]. Its first two cumulants can be obtained by cumulants matching [14]:

$$
\begin{aligned}
& \kappa_{m}\left(A C I_{i}^{k}\right) \\
& =\sum_{\ell \in \mathcal{L}_{i}} \frac{x_{i}^{\ell}}{\left|a_{i}\right|} 2 \pi \widetilde{\mu}_{m}\left(g_{f}\right) \widetilde{\mu}_{m}\left(g_{\theta}\right) p_{s u}^{m}\left(\frac{\gamma_{k, \ell}}{\gamma_{0}}\right)^{m} \int_{d_{0}}^{R_{\epsilon}} g_{d}^{m}(r) r d r \\
& =\sum_{\ell \in \mathcal{L}_{i}} x_{i}^{\ell} \cdot \kappa_{m}\left(a c i_{i}^{k, \ell}\right),
\end{aligned}
$$

where $\widetilde{\mu}_{m}\left(G_{f}\right)$ and $\widetilde{\mu}_{m}\left(G_{\theta}\right)$ are the $m$ th raw moments of the distributions of channel fading and antenna gain, respectively. $d_{0}$ is the minimum separation distance between the TV receiver antenna and the interfering SU. $\kappa_{m}\left(a c i_{i}^{k, \ell}\right)$ is equivalent the cumulant of interference from a single adjacent channel with SU density equal to $1 /\left|a_{i}\right|$.

\section{Optimal Exploitation of Secondary OPPORTUNITY}

From the perspective of the regulator or the operator of the 'TVWS' geo-location database, one way to promote efficient exploitation of spectrum reuse in TV-bands is to admit as many SUs as the aggregated interference constraint would allow. Because the resource sharing among the SUs is typically beyond their control.

To capture the tradeoff between the optimal exploitation of 'TVWS', the interference constraint and the prescribed location probability, we employ Chance Constrained Programming (CCP) [19] to formulate the optimization problem. The CCP formulation can be expressed as follow:

$$
\begin{array}{ll}
\max _{x_{i}^{k}} & \sum_{i=1}^{m} \sum_{\ell \in \mathcal{L}_{i}} x_{i}^{\ell} \\
\text { s.t. } & \operatorname{Pr}\left\{N_{t v}+I_{\mathrm{eff}, i}^{k} \leq \frac{S_{i}^{k}}{\gamma_{0}}\right\} \geq q_{2}^{*}, \\
& 0 \leq \sum_{\ell \in \mathcal{L}_{i}} x_{i}^{\ell} \leq c_{i}, \\
& 0 \leq x_{i}^{\ell} \leq c_{i}^{\prime}, \\
& \forall f_{k} \in \mathcal{F}_{i}, \forall a_{i} \in \mathcal{A} .
\end{array}
$$

Here, the objective is to maximize the total number of admitted SUs over all channels in all pixels. $I_{\mathrm{eff}, i}^{k}$ is a function of the decision variable $x_{i}^{k}$ as defined in the previous section. $c_{i}$ is the maximum number of SUs in pixel $a_{i}$, which depends on the population density, traffic load, etc. $c_{i}^{\prime}$ set the limit on the number of admitted SUs in each channel.

\section{A. Deterministic Equivalent for Interference Constraint}

By Symonds' theorem [20], the probabilistic constraint in (14) can be replaced by a deterministic equivalent, which is generally non-linear and dependent on the distribution of the random coefficients in the constraints.

As discussed earlier, $I_{\text {eff }, i}^{k}$ is a sum of log-normal RVs. By applying Fenton-Wickinson method [21], we can approximate the sum of the aggregate interference and the TV receiver noise (a linear constant) as a single log-normal RV, denoted as $I N_{i}^{k}$. Its first two cumulants are given as

$$
\begin{gathered}
\kappa_{1}\left(I N_{i}^{k}\right)=N_{t v}+\sum_{j \in \mathcal{J}^{k}} x_{j}^{k} \cdot \kappa_{1}\left(c c i_{i, j}^{k}\right)+\sum_{\ell \in \mathcal{L}_{i}} x_{i}^{\ell} \cdot \kappa_{1}\left(a c i_{i}^{k, \ell}\right), \\
\kappa_{2}\left(I N_{i}^{k}\right)=\sum_{j \in \in \mathcal{J}^{k}} x_{j}^{k} \cdot \kappa_{2}\left(c c i_{i, j}^{x}\right)+\sum_{\ell \in \mathcal{L}_{i}} x_{i}^{\ell} \cdot \kappa_{2}\left(a c i_{i}^{k, \ell}\right) .
\end{gathered}
$$

Its distributional parameters, $\mu_{I N_{i}^{k}}$ and $\sigma_{I N_{i}^{k}}^{2}$, can be obtained by [16]

$$
\begin{gathered}
\mu_{I N_{i}^{k}}=\ln \left(\kappa_{1}\left(I N_{i}^{k}\right)\right)-\frac{1}{2} \ln \left[1+\frac{\kappa_{2}\left(I N_{i}^{k}\right)}{\left(\kappa_{1}\left(I N_{i}^{k}\right)\right)^{2}}\right], \\
\sigma_{I N_{i}^{k}}^{2}=\ln \left[1+\frac{\kappa_{2}\left(I N_{i}^{k}\right)}{\left(\kappa_{1}\left(I N_{i}^{k}\right)\right)^{2}}\right] .
\end{gathered}
$$


Together with the log-normal approximation of the TV signal, the probabilistic constraint in (14) can be replaced by a deterministic equivalent as

$$
\begin{aligned}
q^{*} & \leq \operatorname{Pr}\left\{\frac{S_{i}^{k}}{I N_{i}^{k}} \geq \gamma_{0}\right\}=\operatorname{Pr}\left\{\frac{10 \log _{10} S_{i}^{k}}{10 \log _{10} I N_{i}^{k}} \geq 10 \log _{10} \gamma_{0}\right\} \\
& =Q\left(\frac{10 \log _{10}\left(\gamma_{0}\right)+\mu_{I N_{i}^{k}, d B}-\mu_{S_{i}^{k}, d B}}{\sqrt{\sigma_{I N_{i}^{k}, d B}^{2}+\sigma_{S_{i}^{k}, d B}^{2}}}\right),
\end{aligned}
$$

Here the decision variable $x_{i}^{k}$ is included in $\mu_{I N_{i}^{k}, d B}$ and $\sigma_{I N_{i}^{k}, d B}^{2}$ through (15) - (18). And $\mu_{d B}=\frac{10}{\ln 10} \cdot \mu, \sigma_{d B}=$ $\frac{10}{\ln 10} \cdot \sigma$.

\section{B. Linear Approximation of Optimization Problem}

For the large scale scenarios we are typically facing in 'TVWS', solving the optimization problem formulated above directly can be very computationally demanding, as the decision variables are integers and the equivalent deterministic constraint is non-linear.

To simplify the computation, we first approximate the integer decision variable $x_{i}^{k}$ by a continuous one, $\hat{x}_{i}^{k}$. It is a reasonable approximation because $x_{i}^{k}$ can be up to a few thousands in the dense deployment scenarios.

In [19] [22] and [23], several uniformly tighter approximations have been developed to replace the non-linear constraint by a set of linear inequalities. However, these methods are only suited to problems with a small number of random coefficients. Hence, we adopted a simpler approach that has been proven to fit well with typical 'TVWS' scenarios in [9] to linearize the constraint in (19).

First, we rewrite the deterministic constraint (19) by inverting the Q-function

$$
\begin{aligned}
& \mu_{I N_{i}^{k}, d B}-Q^{-1}\left(q_{2}^{*}\right) \sqrt{\sigma_{I N_{i}^{k}, d B}^{2}+\sigma_{S_{i}^{k}, d B}^{2}} \\
& \leq \mu_{S_{i}^{k}, d B}-10 \log _{10}\left(\gamma_{0}\right) .
\end{aligned}
$$

Noting that the non-linearity lies in $\sigma_{I N_{i}^{k}, d B}$, we rewrite (21) as

$$
\begin{aligned}
& \frac{10}{\ln 10} \kappa_{1}\left(I N_{i}^{k}\right) \leq \exp \left[Q^{-1}\left(q_{2}^{*}\right) \sqrt{\sigma_{I N_{i}^{k}, d B}^{2}+\sigma_{S_{i}^{k}, d B}^{2}}\right. \\
& \left.+\frac{1}{2} \sigma_{I N_{i}^{k}, d B}^{2}+\mu_{S_{i}^{k}, d B}-10 \log _{10}\left(\gamma_{0}\right)\right] .
\end{aligned}
$$

Here, the left hand side of the inequality is a linear combination of $\hat{x}_{i}^{k}$ as in (15). And the right hand side, denoted $b_{i}^{k}$, contains the non-linear terms, which we approximate by a linear constant $\hat{b}_{i}^{k}$ :

$$
b_{i}^{k} \approx \hat{b}_{i}^{k}=\exp \left[Q^{-1}\left(q_{2}^{*}\right) \sigma_{S_{i}^{k}, d B}+\mu_{S_{i}^{k}, d B}-10 \log _{10}\left(\gamma_{0}\right)\right] \text {. }
$$

Although the last linear approximation is not uniformly tighter than the original constraint, it is shown in Fig.3 that the approximation error is within $\pm 1 \mathrm{~dB}$ for practical settings. Also, it will be further illustrated in the result section that only few TV channels in a limited area (less than $2 \%$ of total) are around $0.5 \%$ short from the location probability

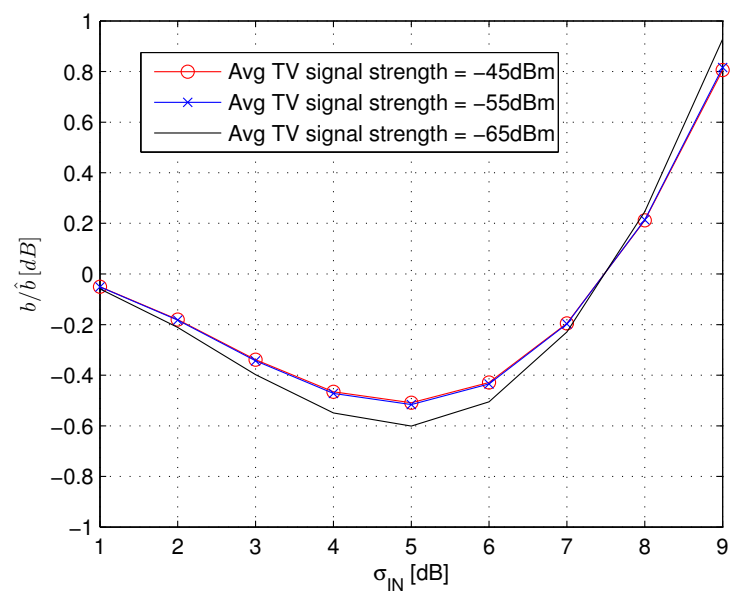

Fig. 3: Ratio between actual constraint and its linear approximation.

requirement due to the approximation error. Thus, it can be easily compensated by applying a small fixed margin to $\hat{b}_{i}^{k}$ if necessary.

\section{Linear Program Formulation}

With some manipulation in the subscripts, we can finally formulate the original optimization problem by the following linear program

$$
\begin{array}{cl}
\max _{X} & 1 X \\
\text { s.t. } & A X \leq B, \\
& 0 \leq H X \leq C, \\
& 0 \preceq X \preceq c^{\prime} .
\end{array}
$$

Here $X$ is the continuous decision variable vector

$$
X=\left(\begin{array}{c}
\hat{x}_{1} \\
\hat{x}_{2} \\
\vdots \\
\hat{x}_{\beta} \\
\vdots
\end{array}\right)
$$

Its cardinality is the sum of the number of available channels in all the pixels, i.e. $|X|=\sum_{i=1}^{m}\left|\mathcal{L}_{i}\right|=\sum_{k=1}^{n}\left|\mathcal{J}^{k}\right|$. Assuming the index of pixel $a_{i}$ is the $\zeta_{i}^{k}$ th elements in the index set $\mathcal{I}^{k}$ and the $\eta_{i}^{k}$ th elements in the index set $\mathcal{J}^{k}$, then $\hat{x}_{i}^{k}$ is mapped to $\hat{x}_{\beta}$ by setting $\beta=\sum_{t=0}^{k-1}\left|\mathcal{J}^{t}\right|+\eta_{i}^{k}$ (Note: $\mathcal{J}^{0}=\emptyset$ ).

The matrix $A$ is the sum of two matrixes, $G^{c c i}$ and $G^{a c i}$, corresponding to the $\kappa_{1}\left(c c i_{i, j}^{k}\right)$ and $\kappa_{1}\left(a c i_{i}^{k, \ell}\right)$ respectively. $G^{c c i}$ is given by

$$
G^{c c i}=\left(\begin{array}{ccccc}
g_{1,1}^{c c i} & g_{1,2}^{c c i} & \cdots & g_{1, \beta}^{c c i} & \cdots \\
g_{2,1}^{c c i} & \ddots & & & \\
\vdots & & \ddots & & \\
g_{\alpha, 1}^{c c i} & & & g_{\alpha, \beta}^{c c i} & \\
\vdots & & & & \ddots
\end{array}\right),
$$


where

$$
g_{\alpha, \beta}^{c c i}= \begin{cases}\kappa_{1}\left(c c i_{i, j}^{k}\right) \quad & \text { if } i \in \mathcal{I}^{k} \text { and } j \in \mathcal{J}^{k} \\ & \alpha=\sum_{t=0}^{k-1}\left|\mathcal{I}^{t}\right|+\zeta_{i}^{k} \\ & \text { and } \beta=\sum_{t=0}^{k-1}\left|\mathcal{J}^{k}\right|+\eta_{j}^{k} \\ & \text { otherwise. }\end{cases}
$$

$G^{a c i}$ has similar structure with its elements $g_{\alpha, \beta}^{a c i}$ given by

$$
g_{\alpha, \beta}^{a c i}= \begin{cases}\kappa_{1}\left(a c i_{i}^{k, \ell}\right), & \text { if } i \in \mathcal{I}^{k} \cap \mathcal{J}^{\ell}, \\ & \alpha=\sum_{t=0}^{k-1}\left|\mathcal{I}^{t}\right|+\zeta_{i}^{k}, \\ & \text { and } \beta=\sum_{t=0}^{\ell-1}\left|\mathcal{J}^{t}\right|+\eta_{i}^{\ell} \\ & \text { otherwise. }\end{cases}
$$

The binary matrix $H$ is used to sum up the number of admitted SUs on all available channels for each pixel. Its elements is given by

$$
h_{\alpha, \beta}= \begin{cases}1, & \alpha=i, \text { and } \beta=\sum_{t=0}^{\ell-1}\left|\mathcal{J}^{t}\right|+\eta_{i}^{\ell}, \forall \ell \in \mathcal{L}_{i} \\ 0, & \text { otherwise }\end{cases}
$$

The constraint vector $B$ and $C$ is given by.

$$
b_{\alpha}=\hat{b}_{i}^{k}, \quad \text { with } \alpha=\sum_{t=0}^{k-1}\left|\mathcal{I}^{t}\right|+\zeta_{i}^{k}, \forall k \in \mathcal{K}_{i}
$$

and

$$
c_{\alpha}=c_{i}, \quad \text { with } \alpha=i .
$$

Once matrixes are constructed, it is straightforward to solve the optimization problem with standard linear program technique, such as, simplex method [25].

\section{NumericAl RESUlts}

We use the linprog function with simplex method in Matlab 2011a [26] to solve the optimization problem formulated above. Then we investigate the relative impact of ACI and $\mathrm{CCI}$ on the optimal solution and the TV reception violation.

\section{A. Sample Study}

For numerical illustration, we consider four identical TV towers regularly deployed in a square area, each broadcasting on a different groups of $10 \mathrm{TV}$ channels out of 40 total TV channels. Wrap around technique [27] is applied to eliminate any border effect (as seen in Fig.4). The TV stations are transmitting with $43 \mathrm{dBW}$ equivalent isotropically radiated power (EIRP) and the mast heights are 200 meters. The TV receiver antenna height is 10 meters, the same as the average surrounding clutter height. The directional rooftop antenna follows specification defined in [28], and is assumed to be pointing towards the closest TV station. With coverage requirement $q_{1}^{*}=0.95$, the radius of the coverage is approximately 40 kilometers. For simplicity, the protection ratio of the TV receiver is assumed to be 'flat' on all adjacent channels, and is equal to $-45 \mathrm{~dB}$ (around the average value of the neighboring 10 channels' protection ratios as defined in Fig.2). The secondary transmitter height is 1.5 meters. The minimum separation distance between the TV antenna and the SU is only limited by their height difference. The secondary user transmit power is $30 \mathrm{dBm}$. The maximum density of SUs is $4000 \mathrm{SUs}$

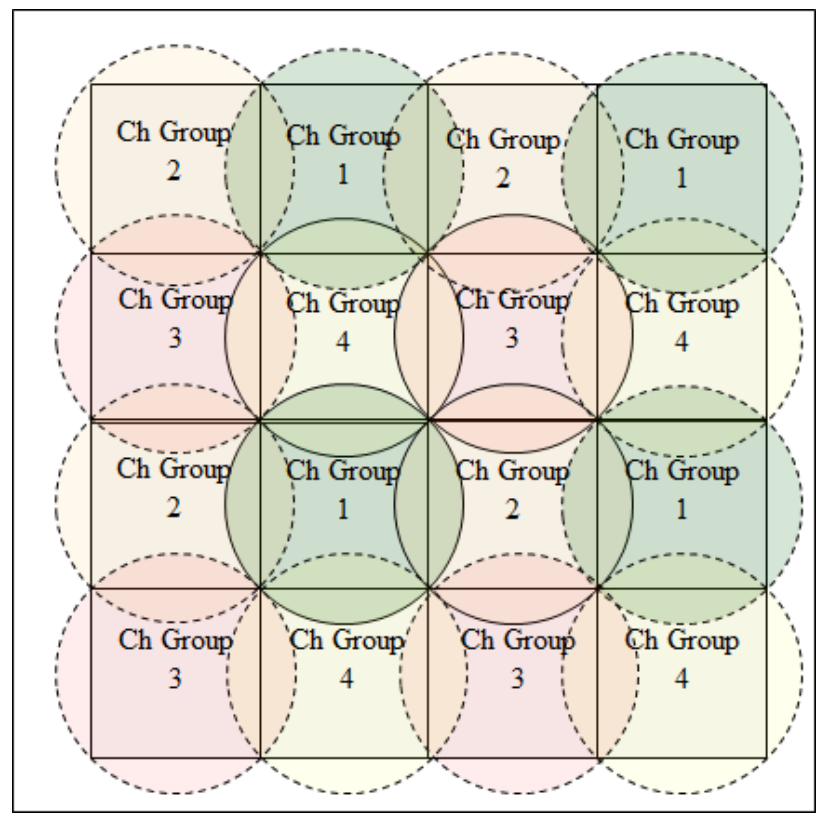

Fig. 4: Illustration of TV tower deployment with wrap around.

per $\mathrm{km}^{2}$ throughout the studied area, and no more 150 SUs per $\mathrm{km}^{2}$ can be admitted to the same channel. With secondary interference, the minimum location probability requirement is $q_{2}^{*}=0.94$. Propagation model ITU-R P1546 [29] is used for TV coverage and CCI link pathloss calculation. Propagation model ITU-R P1411 [30] for over-the-rooftop path is used for ACI link pathloss calculation. The shadow fading standard deviations are $4.65 \mathrm{~dB}$ for TV signal link and $6 \mathrm{~dB}$ for both CCI and ACI links.

The optimal secondary user admission is presented in Fig.5 as a function of distance from the TV station. As can be expected, the optimal solution is achieved by admitting as many SUs as possible in pixels close to the TV station, while only a limited number of SUs are allowed to access TV-bands at the coverage boundary.

For comparison, we also plot the results when either only CCI constraint or ACI constraint is applied. Clearly, ACI constraint is the limiting factor at the area not very far from the TV coverage boundary, as the number of admitted SUs with only ACI constraint matches closely to that under both constraints. However, the spectrum reuse opportunity is overestimated in the region between $10-20 \mathrm{~km}$ away from the TV station if only ACI constraint is applied. On the other hand, with only CCI constraint, $100 \%$ admission rate is reached only at area close to the TV station, but more users are admitted in the middle range of the coverage area than the other two cases.

Monte Carlo simulation is performed to exam the actual TV reception quality with the optimized SU admission. Fig.6 depicts the distribution of the resulting location probabilities of all TV channels in all pixels. The solid line represents the case when the SU admission is optimized over the aggregate interference constraint (21). The protection requirement, $q_{2} \geq$ 


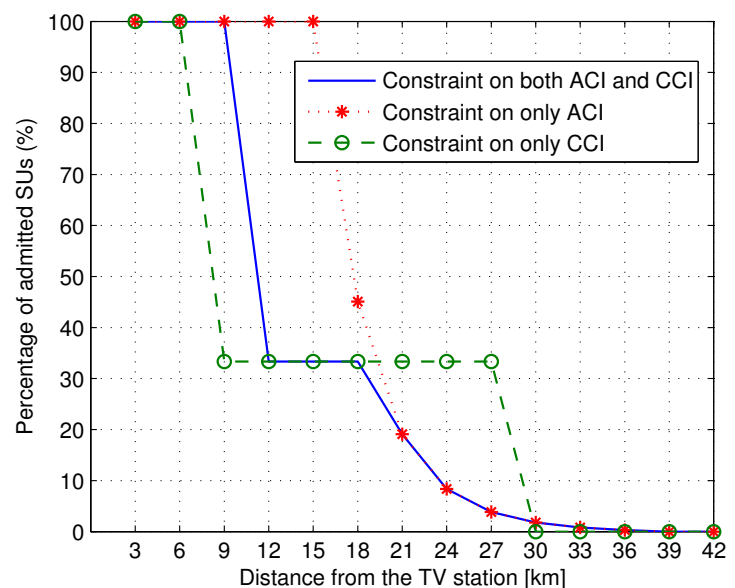

Fig. 5: Percentage of admitted SU at different distance from the TV station.

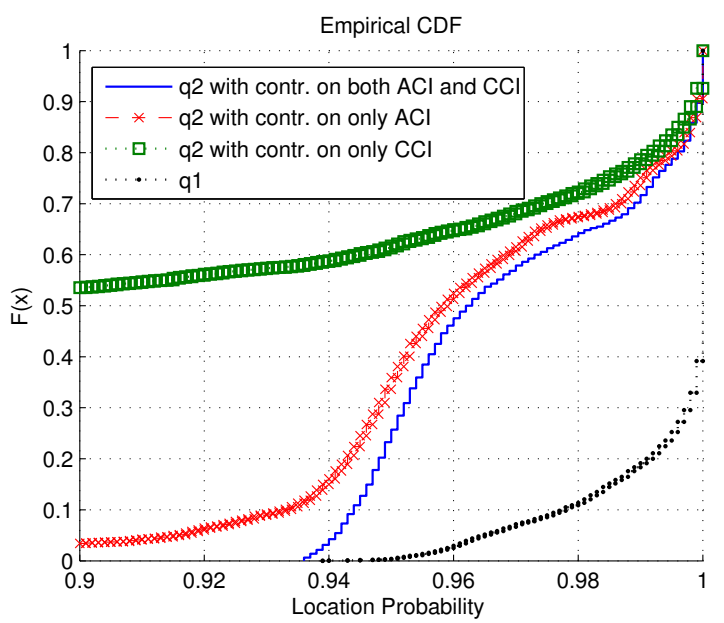

Fig. 6: Location probability distribution over all TV channels in all pixels.

$94 \%$, is satisfied in almost all pixels. A very small portion of the available channels in several pixels experience a location probability slightly lower than the required $94 \%$ due to the approximation error of the interference constraint.

If the SU admission obtained with only CCI constraint is applied into the simulation, the TV reception is severely violated in more than half of the pixels. In comparison, the deterioration in the location probabilities is much less if only ACI constraint is considered. It is evident that the effective interference that causes damage to the TV reception is dominated by ACI.

\section{B. Sensitivity Analysis}

To draw a wider implication, we investigate the effects of two key parameters: the secondary transmit power and the TV receiver protection ratio.

1) Secondary Transmit Power: The optimization problem is solved for three different levels of secondary transmit power: $20 \mathrm{dBm}, 30 \mathrm{dBm}$ and $40 \mathrm{dBm}$, while the rest of the parameters remain the same as in the previous analysis. Fig.7a, shows the average admission ratio in the studied area, i.e., $\sum_{i=1}^{m} \sum_{\ell \in \mathcal{L}_{i}} x_{i}^{\ell} / \sum_{i=1}^{m} c_{i}$. No matter which constraint is applied, less secondary users are admitted as their transmit power increases. Fig.7b shows the average percentage of violated TV channels in all pixels, i.e. $\sum_{i=1}^{m}\left|\mathcal{K}_{i}^{\prime}\right| / \sum_{i=1}^{m}\left|\mathcal{K}_{i}\right|$, where $\mathcal{K}_{i}^{\prime}$ denotes the index set for the set of violated TV channels in pixel $a_{i}$.

Interestingly enough, with optimal SU admission under only CCI constraint, the violation to TV reception actually becomes more severe as the secondary transmit power decreases. At the same time, optimizing over only ACI constraint can achieve a much lower percentage of violated TV channels. In fact, when secondary transmit power is less than $20 \mathrm{dBm}$, the results obtained by optimizing over only ACI constraint are fairly close to that with both constraints. However, if the SU transmit with $40 \mathrm{dBm}$ power, applying either types of constraint would lead to a comparable level of violation.

These results again support our argument that ACI has dominant impact on TV reception violation for the case of dense deployed SUs with limited transmit power. Thus control over ACI alone could achieve near-optimal "TV white spaces" exploitation if such a secondary system is envisaged. In other cases, however, the aggregate interference constraint must take into account both $\mathrm{ACI}$ and $\mathrm{CCI}$ to ensure the protection of TV reception.

2) Protection Ratio: The value of protection ratio used in the previous analysis is based on the average of measurement results from different TV receivers with LTE user equipment being the secondary transmitter. While it is widely used as a reference, the actual protection ratio can vary significantly with different TV receivers and secondary interference signal modulations.

Fig.8 depicts the impact of the different protection ratio values on the secondary reuse opportunity. A "low-end" TV receiver with poor ACI rejection capability is represented by an average protection ratio as high as $-35 \mathrm{~dB}$ [13]. Apparently, the poor TV receiver quality greatly reduced the opportunity for secondary spectrum reuse due to the strong ACI influence on TV reception. For better TV receivers that are commercially available, the protection ratio can reach an average value of $-55 \mathrm{~dB}$. The number of admitted SUs is more than doubled as compare to the case with "low-end" TVs. While ACI still has a stronger influence over the TV reception in this case, the damaging effect of CCI begins to surface and controlling only ACI is no longer sufficient for TV protection (Fig.8b). In fact, $\mathrm{CCI}$ could dominate the aggregate interference if the ACI rejection capability of TV receiver continues to improve. For instance, assuming a "next-generation" TV has an average protection ratio as low as $-75 \mathrm{~dB}$, the number of admitted SUs greatly increases and sufficient protection of the TV reception can be provided by considering CCI constraint only. 


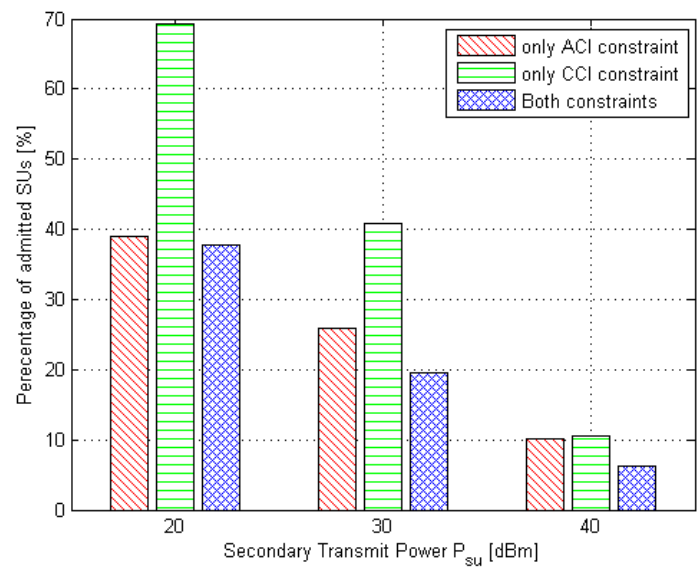

(a) Percentage of admitted SUs in all pixels (\%),

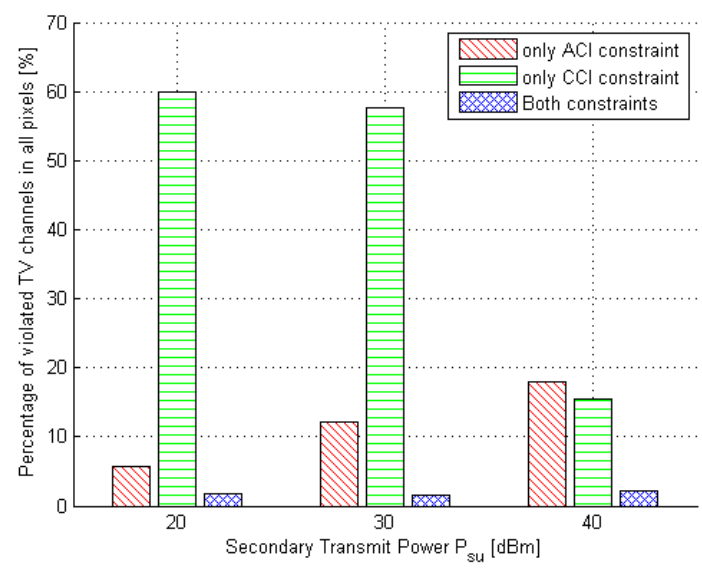

(b) Percentage of violated TV channels in all pixels (\%),

Fig. 7: Effect of different secondary transmit power $p_{s u}$ $\left(\gamma_{k, \ell}=-45 \mathrm{~dB}\right)$.

\section{CONCLUSION}

In this work, we have investigated the 'TV white spaces' opportunity for portable or mobile secondary devices. A new framework for assessing the potential of secondary spectrum reuse in TV-bands is proposed, which to the best of our knowledge is the first framework with combined co-channel and adjacent channel interference constraints. The spectrum reuse opportunity has been assessed in terms of the number of secondary users admitted to each available channel at different locations, by solving an optimization problem with probabilistic interference constraint. With in-depth analysis of the interference characteristic of the optimal solution, we have identified the dominant impact of the aggregate adjacent channel interference on TV reception quality. In particular, for densely deployed portable/mobile secondary devices with a limited transmit power, near-optimal exploitation of the spectrum reuse opportunity can be achieved by controlling only adjacent channel interferences from nearby SUs. Therefore,

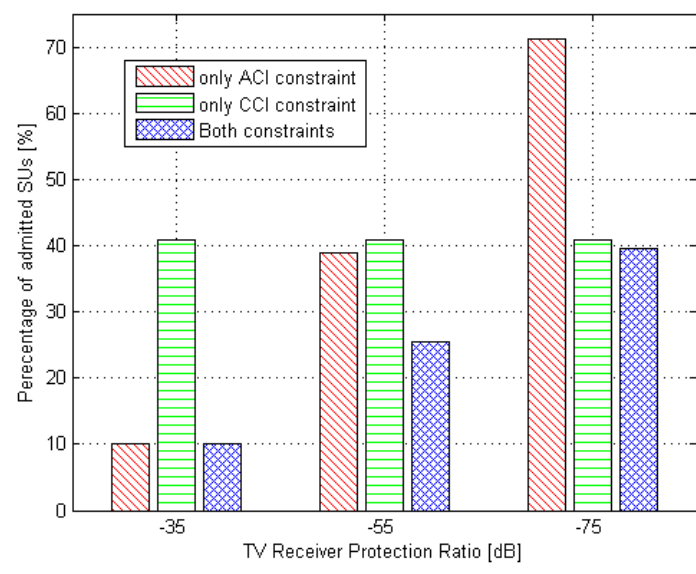

(a) Percentage of admitted SUs in all pixels (\%),

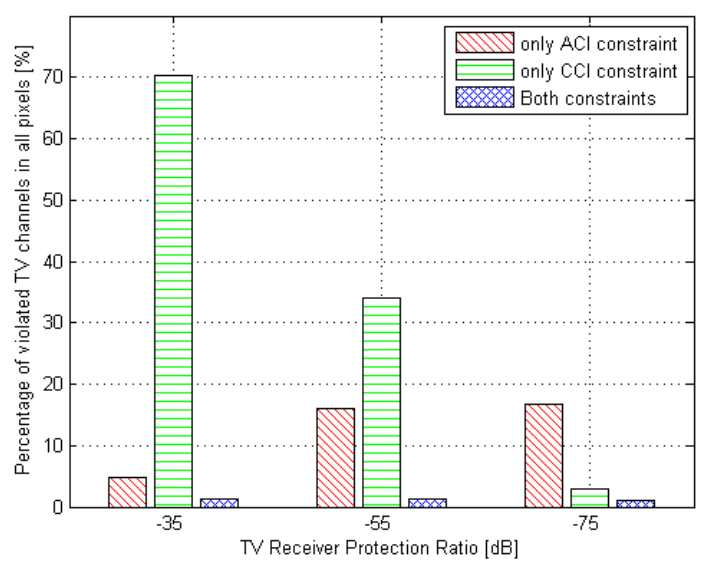

(b) Percentage of violated TV channels in all pixels (\%),

Fig. 8: Effect of different protection ratio $\gamma_{k, \ell}\left(p_{s u}=30 \mathrm{dBm}\right)$.

it is possible to significantly reduce the complexity of the geo-location database by avoiding explicit coordination of cochannel interferences from SUs over a large geographical area. At the same time, we have also showed the deficiency of existing frameworks considering only co-channel interference constraint. It has been proven to provide insufficient protection for the TV reception, unless the adjacent channel interference rejection performance of the TV receiver can be drastically improved.

Overall, we have demonstrated the necessity of incorporating co-channel and adjacent channel interference in the assessment of 'TV white spaces' and established the methodology for that. To extend the framework, we will refine the linear approximation and apply it to evaluate the potential of 'TV white spaces' in a real environment with inhomogeneous user demand distribution and non-flat protection ratios. Another possible direction of extensions is to include multiple secondary systems. 


\section{ACKNOWLEDGMENT}

The research leading to these results has received partial funding from the European Union's Seventh Framework Programme FP7/2007-2013 under grant agreement $n^{\circ} 248303$ (QUASAR). The input and suggestions from the partners in the QUASAR project is gratefully acknowledged.

\section{REFERENCES}

[1] M. Nekovee, "Quantifying the Availability of TV White Spaces for Cognitive Radio Operation in the UK," in IEEE International Conference on Communications Workshops, Jun. 2009.

[2] K. Harrison, S. Mishra, and A. Sahai, "How much white-space capacity is there?," in IEEE International Symposium on Dynamic Spectrum Access Networks (DySPAN), Apr. 2009.

[3] Federal Communications Commission, "In the Matter of Unlicensed Operation in the TV Broadcast Bands: Second Memorandum Opinion and Order," Sept. 2010. [Online] Available: http://www.fcc.gov.

[4] Ofcom, Digital Dividend: Geolocation for Cognitive Access," Nov. 2009, [Online]. Available: http://stakeholders.ofcom.org.uk/consultations/cogaccess/.

[5] ECC Report 159, "Technical and Operational Requirements for the Possible Operation of Coginitive Radio Systems in the White Spaces of the Frequency Band 470-790MHz," Jan. 2011, [Online]. Available: http://www.ero.dk.

[6] ECC Report 148, "Measurements on the Performance of DVB-T Receivers in the Presence of Interference from the Mobile Service (Especially from LTE)", Jan. 2010, [Online]. Available: http://www.ero.dk.

[7] J. van de Beek, J. Riihijarvi, A. Achtzehn, and P. Mahonen, "TV White Space in Europe," IEEE Transactions on Mobile Computing, vol. 11, no. 2, pp. $178-188$, Feb. 2012.

[8] R. Jantti, J. Kerttula, K. Koufos, and K. Ruttik, "Aggregate Interference with FCC and ECC White Space Usage Rules: Case Study in Finland," in IEEE International Symposium on Dynamic Spectrum Access Networks (DySPAN), May 2011.

[9] K. Ruttik, K. Koufos, and R. Jäntti, "Modeling of the Secondary System's Generated Interference and Studying of Its Impact on the Secondary System Design ," Radio Engineering, vol. 18, no. 7, pp. 1271-1278, Dec. 2010.

[10] Y. Selén, J. Kronander, "Optimizaing Power Limits for White Space Devices under a Probability Constraint on Aggregated Inteference," submitted to IEEE International Symposium on Dynamic Spectrum Access Networks (DySPAN), 2012.

[11] E. Obregon, L. Shi, J. Ferrer, and J. Zander, "Experimental Verification of Indoor TV White Space Opportunity Prediction Model," in the Fifth International Conference on Cognitive Radio Oriented Wireless Networks Communications (CROWNCOM), Jun. 2010.

[12] H. Karimi, "Geolocation Databases for White Space Devices in the UHF TV Bands: Specification of Maximum Permitted Emission Levels," in IEEE Symposium on New Frontiers in Dynamic Spectrum Access Networks (DySPAN), May 2011.

[13] E. Obregon, L. Shi, J. Ferrer, and J. Zander, "A Model for Aggregate Adjacent Channel Interference in TV White Space," in 2011 IEEE 73rd Vehicular Technology Conference (VTC Spring), May 2011.

[14] L. Shi, K.W. Sung, and J. Zander, "Controlling Aggregate Interference under Adjacent Channel Interference Constraint in TV White Space," Accepted by 7th International Conference on Cognitive Radio Oriented Wireless Networks and Communications, CROWNCOM '12, Jun. 2012.

[15] QUASAR Deliverable D5.1, "Model Integration and Spectrum Assessment Methodology," March 2011. Available: http://www.quasarspectrum.eu/.

[16] M. Tercero, K. W. Sung, and J. Zander, "Aggregate Interference from Secondary Users with Heterogeneous Density," in IEEE 22nd International Symposium on Personal Indoor and Mobile Radio Communications (PIMRC), 2011.

[17] M. Aljuaid and H. Yanikomeroglu, "Identifying Boundaries of Dominant Regions Dictating Spectrum Sharing Opportunities for Large Secondary Networks," in IEEE 21st International Symposium on Personal Indoor and Mobile Radio Communications (PIMRC), 2010 , Sept. 2010.
[18] A. Ghasemi and E. Sousa, "Interference Aggregation in SpectrumSensing Cognitive Wireless Networks," IEEE Journal of Selected Topics in Signal Processing, , vol. 2, no. 1, pp. 41-56, Feb. 2008.

[19] F. S. Hillier, "Chance-Constrained Programming with 0-1 or Bounded Continuous Decision Variables," Management Science, vol. 14, no. 1, pp. 34-57, 1967.

[20] G. H. Symonds, "Deterministic Solutions for a Class of ChanceConstrained Programming Problems," Operations Research, vol. 15, no. 3, pp. 495-512, 1967.

[21] L. Fenton, "The Sum of Log-Normal Probability Distributions in Scatter Transmission Systems," IRE Transactions on Communications Systems, vol. 8, no. 1, pp. 57-67, Mar. 1960.

[22] Y. Seppala, "Constructing sets of uniformly tighter linear approximations for a chance constraint," Management Science, vol. 17, no. 11, pp. 736-749, 1971.

[23] D. L. Olson and S. R. Swenseth, "A linear approximation for chance-constrained programming," The Journal of the Operational Research Society, vol. 38, no. 3, pp. 261-267, 1987.

[24] K. Koufos, K. Ruttik, and R. Jantti, "Controlling the Interference from Multiple Secondary Systems at the TV Cell Border," in IEEE 22nd International Symposium on Personal Indoor and Mobile Radio Communications (PIMRC), Sept. 2011, pp. $645-649$.

[25] M. S. Bazaraa, J. J. Jarvis, and H. D. Sherali, Front Matter. John Wiley \& Sons, Inc., 2009.

[26] MATLAB, version 7.12.0 (R2011a), Natick, Massachusetts: The MathWorks Inc., 2011.

[27] H. Haas and S. McLaughlin, "A Dynamic Channel Assignment Algorithm for a Hybrid TDMA/CDMA-TDD Interface Using the Novel TS-Opposing Technique," IEEE Journal on Selected Areas in Communications, vol. 19, no. 10, pp. 1831 -1846, oct 2001.

[28] "Directivity and Polarization Discrimination of Antennas in the Reception of Television Broadcasting," ITU-R Recommendation BT.419-3.

[29] "Method for Point-to-Area Predictions for Terrestrial Dervices in the Frequency Range $30 \mathrm{MHz}$ to $3000 \mathrm{MHz}$," ITU Recommendation P.1546-4.

[30] "Propagation Data and Prediction Methods for the Planning of Short-Range Outdoor Radio Communication Systems and Radio Local Area Networks in the Frequency Range $300 \mathrm{MHz}$ to $100 \mathrm{GHz}$,' ITU Recommendation P.1411. 